\title{
Correction
}

\section{Correction: Utevsky et al., "Precuneus Is a Functional Core of the Default-Mode Network"}

In the article, "Precuneus Is a Functional Core of the Default-Mode Network" by Amanda V. Utevsky, David V. Smith, and Scott A. Huettel, which appeared on 932-940 of the January 15, 2014 issue, the left frontoparietal network was mistaken for the right frontoparietal network. This error was referenced in several places and corrected below. These corrections do not affect the main conclusions and interpretation of the paper.

\begin{abstract}
On page 932, in line 9, "Specifically, task performance led to increased connectivity (compared to rest) between the precuneus and the right frontoparietal network ( $\mathrm{rFPN}$ )" was changed to, "Specifically, task performance led to increased connectivity (compared to rest) between the precuneus and the left frontoparietal network (IFPN)".
\end{abstract}

\section{Results}

On page 935, right column, first paragraph, in line 14, the sentence "Of these four networks, the right frontoparietal network (rFPN; Fig. 2A)" was changed to "Of these four networks, the left frontoparietal network (IFPN; Fig. 2A) and default-mode network (DMN; Fig. 2A)".

On page 935, right column, first paragraph, in line 21, "In contrast, the conjunction analysis across the rFPN connectivity maps revealed significant connectivity with the network in the opposite direction-heightened at task compared to rest; this difference was found in precuneal and cuneal cortex (Table 2). The separate conjunctions across the DMN and rFPN connectivity maps revealed one region of overlap within the precuneus (coordinates: $6,-63,42$; Fig. $2 B$ )" was changed to, "In contrast, the conjunction analysis across the lFPN connectivity maps revealed significant connectivity with the network in the opposite direction-heightened at task compared to rest; this difference was found in precuneal and cuneal cortex (Table 2). The separate conjunctions across the DMN and lFPN connectivity maps revealed one region of overlap within the precuneus (coordinates: 6, -63, 42; Fig. 2B)".

On page 935, right column, second paragraph, in first line of the Results section, "To investigate whether the overlap region observed in precuneus exhibited network-specific connectivity, we compared the magnitude of connectivity with DMN and rFPN. We found that the absolute magnitude of connectivity of the precuneus subregion was greater with the DMN than with the rFPN in both the primary and replication groups" was changed to, "To investigate whether the overlap region observed in precuneus exhibited networkspecific connectivity, we compared the magnitude of connectivity with DMN and IFPN. We found that the absolute magnitude of connectivity of the precuneus subregion was greater with the DMN than with the lFPN in both the primary and replication groups".

On page 936, left column, first paragraph under Figure 3, 8 lines down, "this distinct precuneus region exhibited heightened connectivity with DMN compared to rFPN in both the primary and replication groups" was changed to, "this distinct precuneus region exhibited heightened connectivity with DMN compared to IFPN in both the primary and replication groups".

On page 937, left column, second paragraph, line 2, "We investigated this issue and found that the precuneus exhibits state-dependent interactions with both the right-lateralized frontoparietal network (rFPN) and the default-mode network (DMN)." was changed to, "We investigated this issue and found that the precuneus exhibits state-dependent interactions with both the left-lateralized frontoparietal network (IFPN) and the default-mode network (DMN)".

On page 937, right column, fourth paragraph, line 9, "This is crucial, as typical seed-based analyses would have been unable to distinguish the separate responses from DMN and rFPN in our study" was changed to, "This is crucial, as typical seed-based analyses would have been unable to distinguish the separate responses from DMN and IFPN in our study".

On page 938, left column, first paragraph, line 9, "While our study demonstrated that the precuneus exhibits task-dependent connectivity with rFPN and DMN" was changed to, "While our study demonstrated that the precuneus exhibits task-dependent connectivity with lFPN and DMN". Line 22, last sentence, “. . . to gain further insight into the functional dynamics of the rFPN and DMN." was changed to, ". . . to gain further insight into the functional dynamics of the lFPN and DMN."

\section{Figure 2}

On page 935, Figure $2 A$, "X $=3$ " was changed to " $\mathrm{X}=-41$ " and Figure $2 B$, "Coactivation with rFPN" was changed to "Coactivation with lFPN".

On page 935, in the caption to Figure 2: “ $A$, We identified two networks that exhibit significant connectivity differences in the task and rest states, the default-mode network (top) and the right frontoparietal network (bottom). Activations shown are the intersections of 
each z-transformed network map, respectively, across the six groups examined. $\boldsymbol{B}$, Areas within precuneus exhibited task-dependent connectivity with rFPN (red) and DMN (blue)." was changed to " $A$, We identified two networks that exhibit significant connectivity differences in the task and rest states, the default-mode network (top) and the left frontoparietal network (bottom). Activations shown are the intersections of each $\mathrm{z}$-transformed network map, respectively, across the six groups examined. $\boldsymbol{B}$, Areas within precuneus exhibited task-dependent connectivity with IFPN (red) and DMN (blue).”

\section{Figure 3}

On p. 936, Figure 3A, "Coactivation with rFPN" was changed to "Coactivation with lFPN".

On page 936, in the caption to Figure 3, "We examined connectivity between peak voxels of canonical default-mode network (DMN) regions (Smith et al., 2009) and our rest $>$ task and task>rest connectivity maps for both the DMN and right frontoparietal (rFPN) networks. A, Parameter estimates quantifying subject-specific rest-minus-task connectivity between the selected region and the DMN (blue) and rFPN (red) in both the primary groups of each task and the replication groups of each task." was changed to, "We examined connectivity between peak voxels of canonical default-mode network (DMN) regions (Smith et al., 2009) and our rest>task and task>rest connectivity maps for both the DMN and left frontoparietal (lFPN) networks. A, Parameter estimates quantifying subjectspecific rest-minus-task connectivity between the selected region and the DMN (blue) and IFPN (red) in both the primary groups of each task and the replication groups of each task."

\section{Table 2}

On p. 935, right column, the title of Table 2, "State Differences in Connectivity with Right Frontoparietal Network" was changed to "State Differences in Connectivity with Left Frontoparietal Network".

On p. 935, right column, in the caption to Table 2, "Regions whose connectivity with the rFPN was greater in the task state compared to the resting state, after correcting for two-tailed tests across the four independent networks" was changed to "Regions whose connectivity with the IFPN was greater in the task state compared to the resting state, after correcting for two-tailed tests across the four independent networks".

A

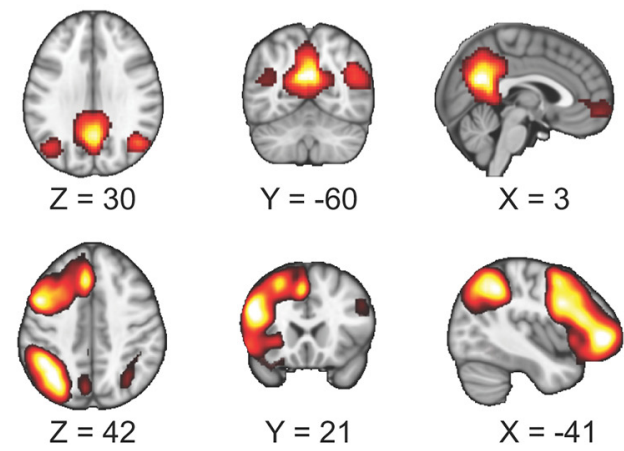

B

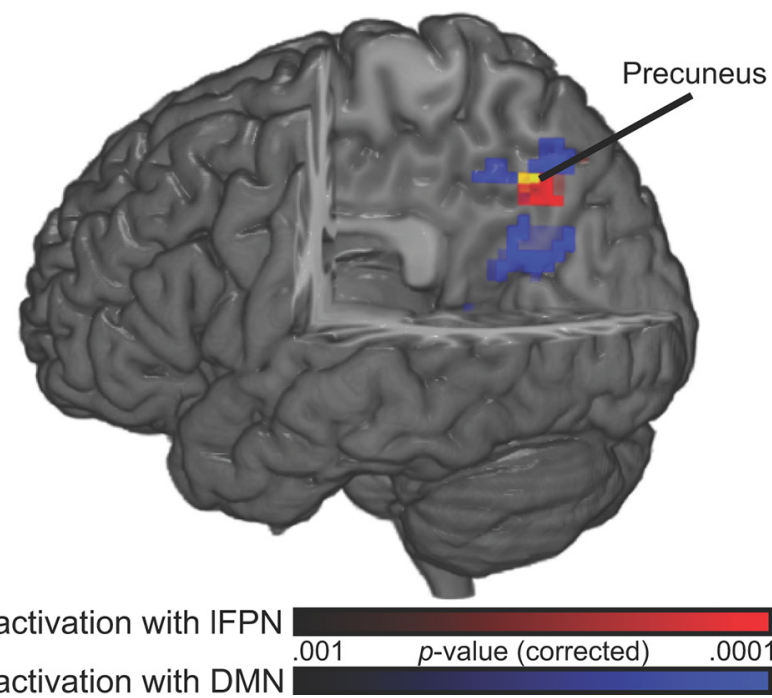

Figure 2. 


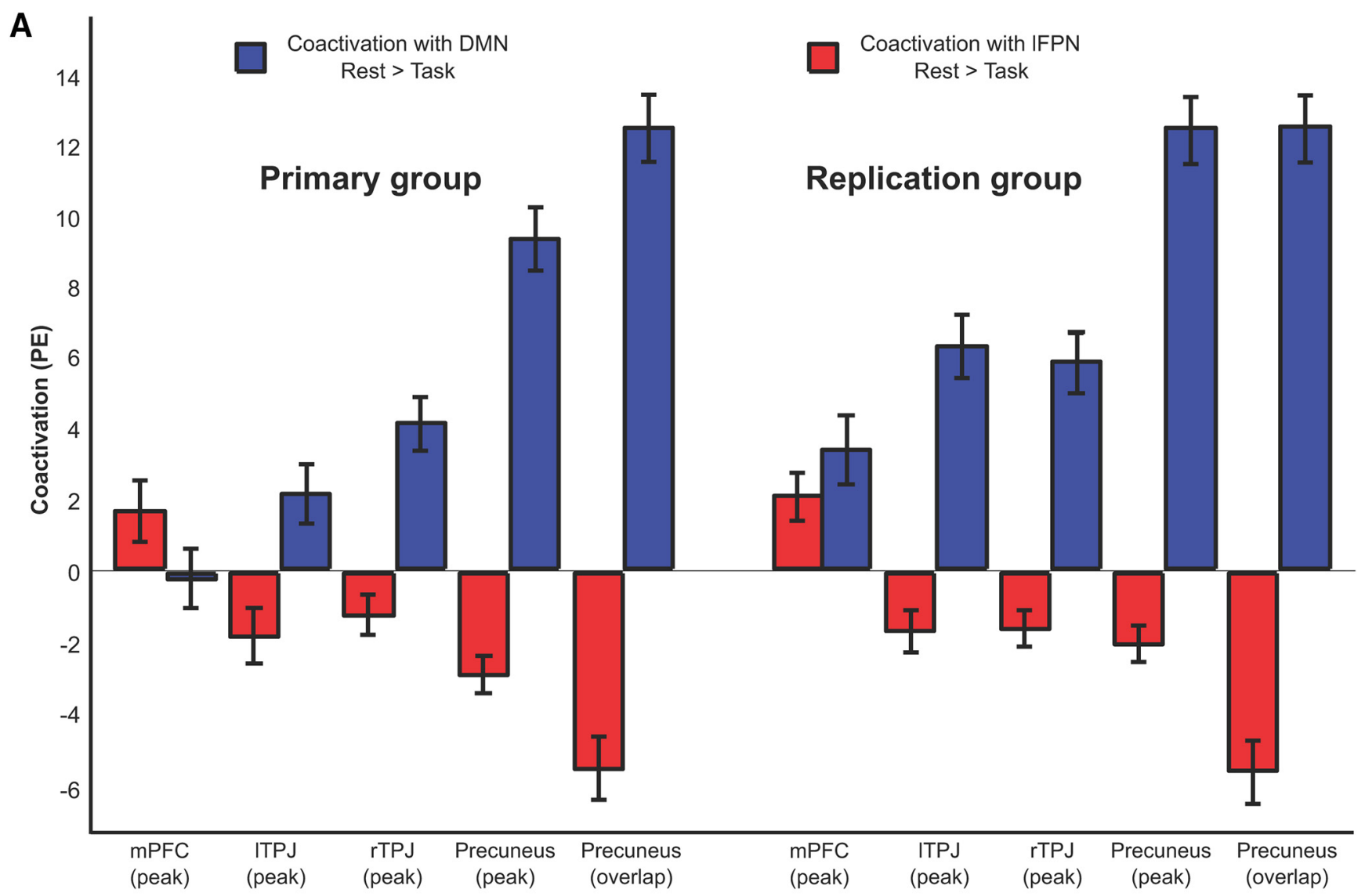

B

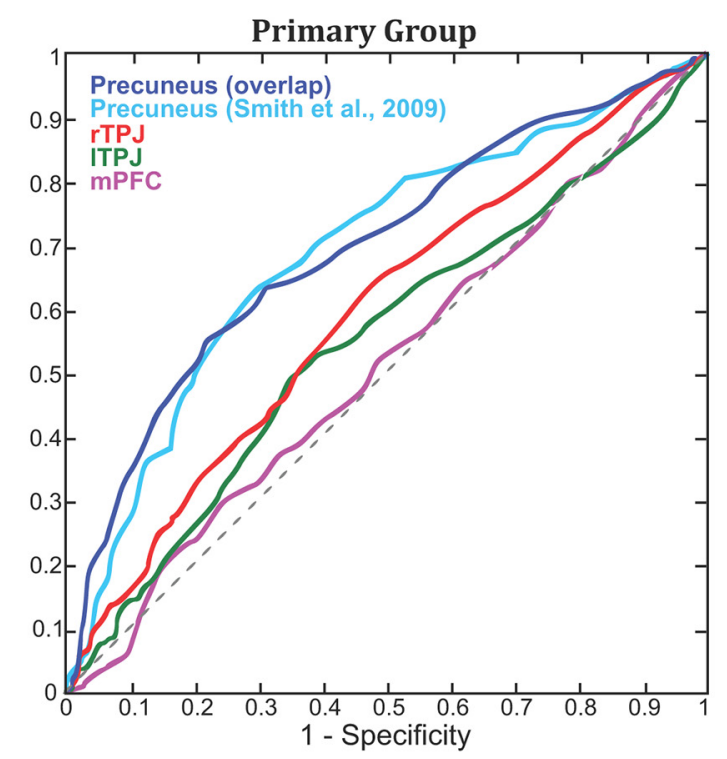

Cluster Region

ctivation with IFPN

Rest > Task

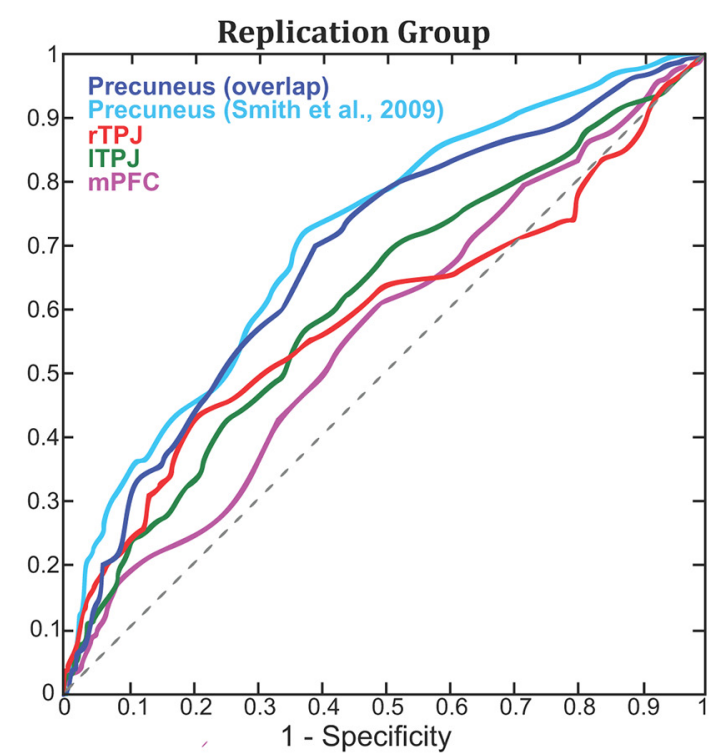

Figure 3. 